\title{
Tomograms and other transforms: A unified view
}

\author{
M. A. Man'ko*†, V. I. Man'ko*†and R. Vilela Mendes ${ }^{\ddagger \dagger}$
}

\begin{abstract}
A general framework is presented which unifies the treatment of wavelet-like, quasidistribution, and tomographic transforms. Explicit formulas relating the three types of transforms are obtained.

The case of transforms associated to the symplectic and affine groups is treated in some detail. Special emphasis is given to the properties of the scale-time and scale-frequency tomograms. Tomograms are interpreted as a tool to sample the signal space by a family of curves or as the matrix element of a projector.
\end{abstract}

\section{Introduction}

Several types of integral transforms [1] [2] are used for signal processing in physics, engineering, medicine, etc. In addition to the traditional Fourier analysis [3], wavelet analysis has been extensively developed in the last two decades [4] [5] [6]. These two types of transforms are linear transforms. In addition, the Wigner-Ville quasidistribution [7] [8], a bilinear transform, provides optimal energy resolution in the joint time-frequency domain. A joint time-frequency description of signals is important, because in many applications (biomedical, seismic, radar, etc.) the signals are of finite (sometimes

${ }^{*}$ P. N. Lebedev Physical Institute, Leninskii Prospect 53, Moscow 117924, Russia, email: mmanko@sci.lebedev.ru

†Zentrum für interdisziplinäre Forschung, Universität Bielefeld, Wellenberg 1, 33615 Bielefeld, Germany

${ }^{\ddagger}$ Grupo de Física Matemática, Complexo Interdisciplinar, Universidade de Lisboa, Av. Gama Pinto, 2 - P1699 Lisboa Codex, Portugal, e-mail: vilela@cii.fc.ul.pt 
very short) duration. However, the oscillating cross-terms in the WignerVille quasidistribution make the interpretation of this transform a difficult matter. Even if the average of the cross-terms is very small, their amplitude may be greater than the signal terms in time-frequency regions that carry no physical information. To profit from the time-frequency energy resolution of the bilinear transforms while controlling the cross-terms problem, modifications to the Wigner-Ville transform have been proposed. Transforms in the Cohen class [9] make a two-dimensional filtering of the Wigner-Ville quasidistribution and the Gabor spectrogram [10] is a truncated version of this quasidistribution.

Recently, a new type of strictly positive bilinear transforms have been proposed, namely, the Radon-Wigner transform [11] [12] or, more generally, the noncommutative tomography [13] which, in addition to the timefrequency domain, also applies to other noncommutative pairs like time-

scale, frequency-scale, etc. It is this last class of transforms that will be called the tomograms in this paper. The tomograms are strictly positive probability densities, provide a full characterization of the signal and are robust in the presence of noise.

Developing a general operator scheme, we show how linear transforms like Fourier transform or wavelets are related to the quasidistributions and the tomograms. Explicit general formulas are derived relating the three types of transforms. The time-frequency plane is then briefly discussed because most of the material has been treated before [13. Special emphasis is, however, given to the transforms associated to the affine group, namely, to the time-

scale and frequency-scale tomograms. To clarify the physical meaning of the tomograms, we also propose an interpretation as a sampling of the signal space by families of curves or as the action of a projection operator.

\section{Wavelet-like transforms, quasidistributions, and tomograms}

We present a unified general construction of three types of transforms used in signal analysis. The first class consists of wavelet-type transforms, the second of quasidistributions, and in the third class are the tomographic transforms. Quasidistributions are transforms like the Wigner-Ville one [7] [8] or the P- 
quasidistributions of Glauber and Sudarshan [14] [15]. Husimi-Kano positive quasidistributions [16] [17] will be discussed as well. These types of quasidistributions are unified in the class of $s$-ordered quasidistributions [18].

In quantum mechanics, quasidistributions describe a quantum state in terms of phase-space quasiprobability densities. In signal analysis, quasidistributions describe the structure of analytic signals in the time-frequency plane. There also exist quasidistributions characterizing the signal structure in the time-scale plane [19] [20] [21] [22]. We refer to quasiprobability densities because the corresponding functions are not conventional probabilities, being either complex or nonpositive. In the case of positive quasiprobabilities like the Husimi-Kano function, the two arguments of the function are not simultaneously measurable random variables. The corresponding observables do not commute and the uncertainty relation prevents the existence of a joint distribution function for noncommuting observables. Time $\hat{t}$ and frequency $\hat{\omega}$, time and scale $\frac{1}{2}(\hat{\omega} \hat{t}+\hat{t} \hat{\omega})$, or frequency and scale are common examples of such pairs of noncommuting observables.

The general setting for our construction is as follows.

Signals $f(t)$ are considered to be vectors $|f\rangle$ belonging to a dense nuclear subspace $\mathcal{N}$ of a Hilbert space $\mathcal{H}$ with dual space $\mathcal{N}^{*}$ (and the canonical identification $\left.\mathcal{N} \subset \mathcal{N}^{*}\right) .\{U(\alpha): \alpha \in I\}$ is a family of operators defined on $\mathcal{N}^{*}$, and a fortriori on $\mathcal{N}$ by the canonical identification $\mathcal{N} \subset \mathcal{N}^{*}$. In many cases, the family of operators $U(\alpha)$ generates a unitary group. However, this is not a necessary condition for the consistency of the formalism, provided the completeness conditions discussed below are satisfied.

In this setting, three types of transforms are defined. Consider a reference vector $h \in \mathcal{N}^{*}$ chosen in such a way that the linear span of $\left\{U(\alpha) h \in \mathcal{N}^{*}: \alpha \in I\right\}$ is dense in $\mathcal{N}^{*}$. This means, in particular, that, out of the set $\{U(\alpha) h\}$, a complete set of vectors can be chosen to serve as a basis. Two of the transforms considered are:

$$
\begin{gathered}
W_{f}^{(h)}(\alpha)=\langle U(\alpha) h \mid f\rangle, \\
Q_{f}(\alpha)=\langle U(\alpha) f \mid f\rangle .
\end{gathered}
$$

If $U(\alpha)$ is a unitary operator generated by $B(\vec{\alpha})=\alpha_{1} t+i \alpha_{2} \frac{d}{d t}$ and $h$ is a (generalized) eigenvector of the time-translation operator, $W_{f}^{(h)}(\alpha)$ becomes a Fourier transform. With the same $B(\vec{\alpha})$ plus the parity operator, $Q_{f}(\alpha)$ would be the Wigner-Ville transform. Similarly, for $B(\vec{\alpha})=\alpha_{1} D+i \alpha_{2} \frac{d}{d t}$, 
where $D$ is the dilation operator $D=-\frac{1}{2}\left(i t \frac{d}{d t}+i \frac{d}{d t} t\right), W_{f}^{(h)}(\alpha)$ is a wavelet transform and $Q_{f}(\alpha)$ the Bertrand transform.

We will denote the transforms of the $W_{f}^{(h)}$-type as wavelet-type transforms and those of the $Q_{f}$-type as quasidistribution transforms.

In general, if $U(\alpha)$ are unitary operators, by Stone's theorem, there are self-adjoint operators $B(\alpha)$ such that

$$
\begin{aligned}
& W_{f}^{(h)}(\alpha)=\left\langle h\left|e^{i B(\alpha)}\right| f\right\rangle, \\
& Q_{f}^{(B)}(\alpha)=\left\langle f\left|e^{i B(\alpha)}\right| f\right\rangle .
\end{aligned}
$$

In this case, because $B(\alpha)$ has a real valued spectrum, another transform may be defined, namely,

$$
M_{f}^{(B)}(X)=\langle f|\delta(B(\alpha)-X)| f\rangle .
$$

This is what we call the tomographic transform or tomogram. In contrast to the quasiprobabilities, the transform $M_{f}^{(B)}(\alpha)$ is positive and, as we will see below, it can be correctly interpreted as a probability distribution. Therefore, it benefits from the properties of the bilinear transforms, without being plagued by the interpretation ambiguities associated to the quasidistribution transforms.

For a normalized vector $|f\rangle$,

$$
\langle f \mid f\rangle=1
$$

the tomogram is a normalized function

$$
\int M_{f}^{(B)}(X) d X=1
$$

and therefore, it may be interpreted as a probability distribution for the random variable $X$ corresponding to the observable defined by the operator $B(\mu)$. The tomogram is a homogeneous function

$$
M_{f}^{(B / p)}(X)=|p| M_{f}^{(B)}(p X) .
$$

The three classes of transforms are mutually related

$$
M_{f}^{(B)}(X)=\frac{1}{2 \pi} \int Q_{f}^{(k B)}(\alpha) e^{-i k X} d k
$$


and

$$
Q_{f}^{(B)}(\alpha)=\int M_{f}^{(B / p)}(X) e^{i p X} d X
$$

Wavelet-type transforms, quasidistributions, and tomograms are related by the formulas

$$
\begin{gathered}
Q_{f}^{(B)}(\alpha)=W_{f}^{(f)}(\alpha), \\
W_{f}^{(h)}(\alpha)=\frac{1}{4} \int e^{i X}\left[M_{f_{1}}^{(B)}(X)-i M_{f_{2}}^{(B)}(X)-M_{f_{3}}^{(B)}(X)+i M_{f_{4}}^{(B)}(X)\right] d X,
\end{gathered}
$$

where

$$
\begin{array}{r}
\left|f_{1}\right\rangle=|h\rangle+|f\rangle ; \quad\left|f_{3}\right\rangle=|h\rangle-|f\rangle \\
\left|f_{2}\right\rangle=|h\rangle+i|f\rangle ; \quad\left|f_{4}\right\rangle=|h\rangle-i|f\rangle .
\end{array}
$$

Another important case concerns operators $U(\mu)$, which can be represented in the form

$$
U(\alpha)=e^{i b(\alpha)} P_{h} e^{-i b(\alpha)},
$$

$P_{h}$ being a projector on a reference vector $|h\rangle$. This creates a quasidistribution of the Husimi-Kano type

$$
H_{f}^{(b)}(\alpha)=\langle f|U(\alpha)| f\rangle .
$$

In the following sections, we show how known examples of wavelet-like and quasidistribution transforms are described within the framework presented above. We will consider quasidistributions such as Wigner-Ville [7] [8], Bertrand [19], and Husimi-Kano [16] [17]. We reformulate the standard wavelet analysis in terms of this general scheme using an operator $U(\vec{\alpha})$ belonging to the two-dimensional affine subgroup of the symplectic group $I S p(2, R)$. Tomographic transform schemes for time-frequency, time-scale, and frequency-scale pairs [13] will be studied within the framework of the general approach. Inversion formulas are obtained for the tomograms as well as the explicit connection of the wavelet transform to the time-scale and frequency-scale tomograms.

\section{Time-frequency transforms}

Here we discuss the case where the operator $B(\vec{\alpha}), \vec{\alpha}=(\mu, \nu)$ is

$$
B^{(S)}(\alpha)=\mu \hat{t}+\nu \hat{\omega}
$$


(with $\hat{\omega}=-i \partial / \partial t$ ) or is equal to this one plus a parity operator. The waveletlike transform in this case is just the Fourier transform and we discuss only the tomograms and the quasidistributions.

The tomogram, that is,

$$
M_{f}^{(S)}(X, \mu, \nu)=\langle f|\delta(\mu \hat{t}+\nu \hat{\omega}-X)| f\rangle
$$

was shown in $\|13\|$ to be

$$
M_{f}^{(S)}(X, \mu, \nu)=\frac{1}{2 \pi|\nu|}\left|\int \exp \left[\frac{i \mu t^{2}}{2 \nu}-\frac{i t X}{\nu}\right] f(t) d t\right|^{2} .
$$

The tomogram (15) is normalized if $\langle f \mid f\rangle=1$

$$
\int M_{f}^{(S)}(X, \mu, \nu) d X=1
$$

From the tomogram $M_{f}^{(S)}(X, \mu, \nu)$, the signal $f(t)$ may be recovered up to a phase

$$
f(t) f^{*}(0)=\frac{1}{2 \pi} \int M_{f}^{(S)}(X, \mu, t) \exp \left[i\left(X-\mu \frac{t}{2}\right)\right] d X d \mu .
$$

According to the general scheme, the corresponding quasidistribution is

$$
Q_{f}^{(S)}(\mu, \nu)=\left\langle f\left|e^{i B^{(S)}(\mu, \nu)}\right| f\right\rangle=\int M_{f}^{(S)}(X, \mu, \nu) e^{i X} d X
$$

or

$$
Q_{f}^{(S)}(\mu, \nu)=\int f^{*}\left(t-\frac{\nu}{2}\right) f\left(t+\frac{\nu}{2}\right) e^{i \mu t} d t .
$$

This quasidistribution is called the ambiguity function in the signal processing literature [23].

The tomogram (15) and this quasidistribution are related by Eqs. (9) and (10). The tomogram (15) is also related to another quasidistribution, namely to the Wigner-Ville quasidistribution $W V(\tau, \omega)$ [7] [8] by

$$
M_{f}^{(S)}(X, \mu, \nu)=\int \exp [-i k(X-\mu \omega-\nu \tau)] W V(\tau, \omega) \frac{d k d \omega d \tau}{(2 \pi)^{2}} .
$$

The Wigner-Ville quasidistribution is given by the formula

$$
W V(\tau, \Omega)=\int f\left(\tau+\frac{u}{2}\right) f^{*}\left(\tau-\frac{u}{2}\right) e^{-i \Omega u} d u .
$$


The unitary operator $U(\tau, \Omega)$, which determines the Wigner-Ville quasidistribution by

$$
W V(\tau, \Omega)=\left\langle f\left|U^{(W V)}(\tau, \Omega)\right| f\right\rangle
$$

is

$$
U^{(W V)}(\tau, \Omega)=e^{2 i(\Omega \hat{t}-\tau \hat{\omega})} e^{i \pi\left(\hat{t}^{2}+\hat{\omega}^{2}-1\right) / 2},
$$

the generator being

$$
B^{(W V)}(\tau, \Omega)=2 \tau \hat{\omega}-2 \Omega \hat{t}+\frac{\pi\left(\hat{t}^{2}+\hat{\omega}^{2}-1\right)}{2} .
$$

\section{Wavelets and quasidistributions in the affine group}

\subsection{Wavelets}

The wavelet transform of a signal $f(t)$ is a linear integral transform decomposing the signal into a set of basis functions

$$
W_{f}^{(A)}(s, \tau)=\int f(t) h_{s, \tau}^{*}(t) d t .
$$

The wavelets $h_{s, \tau}(t)$ are kernel functions generated from a basic wavelet $h(\tau)$ by means of a translation and a rescaling $(-\infty<\tau<\infty, s>0)$ :

$$
h_{s, \tau}(t)=\frac{1}{\sqrt{s}} h\left(\frac{t-\tau}{s}\right) \text {. }
$$

Using the operator

$$
U^{(A)}(\tau, s)=\exp (i \tau \hat{\omega}) \exp (i \log s D)
$$

where

$$
D=\frac{1}{2}(\hat{t} \hat{\omega}+\hat{\omega} \hat{t})=\hat{\omega} \hat{t}+\frac{i}{2}, \quad \text { with } \quad \hat{\omega}=-i \frac{\partial}{\partial t},
$$

equation (25) can be represented in the form

$$
h_{s, \tau}(t)=U^{(A) \dagger}(\tau, s) h(t) .
$$


For normalized $h(t)$ the wavelets $h_{s, \tau}(t)$ satisfy the normalization condition

$$
\int\left|h_{s, \tau}(t)\right|^{2} d t=1
$$

The basic wavelet (reference vector) may have different forms, for example,

$$
h(t)=\frac{1}{\sqrt{\pi}} e^{i \omega_{0} t} e^{-t^{2} / 2},
$$

or

$$
h(t)=\left(1-t^{2}\right) e^{-t^{2} / 2}
$$

called the Mexican hat wavelet.

The inverse of the wavelet transform $W_{f}^{(A)}(s, \tau)$ is

$$
f(t)=N_{h}^{-1} \int W_{f}^{(A)}(s, \tau) \frac{1}{\sqrt{s}} h\left(\frac{t-\tau}{s}\right) \frac{d \tau d s}{s^{2}},
$$

with

$$
N_{h}=\int \frac{|H(\omega)|^{2}}{|\omega|} d \omega, \quad H(\omega)=\int h(t) e^{-i \omega t} d t .
$$

One has the property

$$
\int\left|W_{f}^{(A)}(s, \tau)\right|^{2} \frac{d \tau d s}{s^{2}}=N_{h} \int|f(t)|^{2} d t .
$$

Let us consider the operator (26), with the parameters $\vec{\mu}=\left(\mu_{1}, \mu_{2}\right) \equiv(\mu, \nu)$ being

$$
\nu=\log s, \quad \mu=\frac{\tau \log s}{s-1} .
$$

We obtain for the unitary operator (26)

$$
U^{(A) \dagger}(\tau, s) \equiv U^{(A)}(\mu, \nu)=\exp (i \mu \hat{\omega}+i \nu D),
$$

and the operator $B(\vec{\alpha})$ becomes

$$
B_{1}^{(A)}=\mu \hat{\omega}+\nu D .
$$

According to the general scheme with the operator (36), the wavelet transform (24) can be rewritten in the form (11)

$$
W_{f}^{(A)}(s, \tau)=\left\langle h\left|U^{(A)}(\mu, \nu)\right| f\right\rangle .
$$


The commutation relation for the operators $\hat{\omega}$ and $D$ is

$$
[\hat{\omega}, D]=-i \hat{\omega}
$$

The commutation relations (38) define the Lie algebra of the affine group. Therefore, the wavelet transform is the nondiagonal matrix element of a unitary irreducible representation of the affine group. The parameters $\mu$ and

$\nu$ are the group parameters and the Hermitian operator $B_{1}^{(A)}$ belongs to the Lie algebra of the affine group.

\subsection{Quasidistributions}

The diagonal matrix elements of the irreducible representation determine a quasidistribution for the signal $f(t)$

$$
Q_{f}^{(A)}(s, \tau)=\left\langle f\left|e^{i(\mu \hat{\omega}+\nu D)}\right| f\right\rangle,
$$

with $\mu$ and $\nu$ expressed in terms of shift and scaling parameters by Eq. (34).

Defining the action on the vector $|f\rangle$ as

$$
e^{i(\mu \hat{\omega}+\nu D) / 2} f(t)=F(\mu, \nu, t)
$$

the quasidistribution (39) may be rewritten

$$
Q_{f}^{(A)}(s, \tau)=\int F^{*}(-\mu,-\nu, t) F(\mu, \nu, t) d t .
$$

Using the known kernel (Green function) of the operator (35) 24], one obtains for the quasidistribution (41) the following expression in terms of the parameters $\tau$ and $s$ :

$$
Q_{f}^{(A)}(s, \tau)=\int f^{*}\left(\frac{t-\tau / 2}{\sqrt{s}}\right) f(\sqrt{s}[t+\tau / 2]) d t .
$$

The wavelet transform (24) may also be written in a similar form

$$
W_{f}^{(A)}(s, \tau)=\int h^{*}\left(\frac{t-\tau / 2}{\sqrt{s}}\right) f(\sqrt{s}[t+\tau / 2]) d t
$$

as follows from Eq. (11). 


\subsection{Relation of wavelets to time-frequency tomograms}

In view of (31) and (15), one relates the wavelet transform to the timefrequemcy tomogram

$$
\begin{aligned}
& M_{f}^{(S)}(X, \mu, \nu)=\frac{1}{2 \pi|\nu|\left|N_{h}\right|^{2}} \mid \int W_{f}^{(A)}(s, \tau) \frac{h(t)}{|s| \sqrt{s}} \\
& \times\left.\exp \left\{\frac{i \mu}{2 \nu}\left(s^{2} t^{2}+2 s t \tau+\tau^{2}\right)-\frac{i X}{\nu}(s t+\tau)\right\} d s d t d \tau\right|^{2} .
\end{aligned}
$$

The inverse transform reads

$$
\begin{aligned}
W_{f}^{(A)}(s, \tau)= & \frac{\sqrt{s}}{2 \pi \mathcal{D}} \int h^{*}(t) M_{f}^{(S)}(X, \mu, s t+\tau) \\
& \times \exp \left\{i X-\frac{i \mu(s t+\tau)}{2}\right\} d X d \mu d t
\end{aligned}
$$

where

$$
\mathcal{D}=\left[\frac{1}{2 \pi} \int M_{f}^{(S)}(X, \mu, 0) e^{i X} d X d \mu\right]^{1 / 2}
$$

For the Mexican hat wavelet (30), with the admissibility condition

$$
\int h(t) d t=0
$$

one has the explicit form

$$
M_{f}^{(S)}(X, \mu, \nu)=\frac{1}{2 \pi|\nu|\left|N_{h}\right|^{2}}\left|\int W_{f}^{(A)}(s, \tau) K_{M}(s, \tau, \mu, \nu, X) d s d \tau\right|^{2},
$$

where

$$
\begin{aligned}
K_{M}(s, \tau, \mu, \nu, X)= & \sqrt{2 \pi}\left(s-\frac{i \mu s^{3}}{\nu}\right)^{-3 / 2}\left[\frac{(s \mu \tau-s X)^{2}}{\nu^{2}-i \mu \nu s^{2}}-\frac{i \mu s^{2}}{\nu}-1\right] \\
& \times \exp \left[-\frac{(s \mu \tau-s X)^{2}}{2\left(\nu^{2}-i \mu \nu s^{2}\right)}+\frac{i \mu \tau^{2}}{2 \nu}-\frac{i X \tau}{\nu}\right]
\end{aligned}
$$

and

$$
N_{h}=\int|\omega|^{3} e^{-\omega^{2}} d \omega=1 .
$$


The inverse transform for the Mexican hat wavelet reads

$$
W_{f}^{(A)}(s, \tau)=\int R(s, \tau, X, \mu, \nu) M_{f}^{(S)}(X, \mu, \nu) d X d \mu d \nu
$$

the kernel being

$$
R(s, \tau, X, \mu, \nu)=\frac{1}{2 \pi \mathcal{D} \sqrt{s}}\left[1-\left(\frac{\tau-\nu}{s}\right)^{2}\right] \exp \left[-\frac{i}{2}\left(\frac{\nu-\tau}{s}\right)^{2}-\frac{i \mu \nu}{2}+i X\right] .
$$

\subsection{Tomograms. Frequency-scale and time-scale}

The tomogram associated to the operator $B_{1}^{(A)}=\mu \hat{\omega}+\nu D$ has been computed in 13 . It is

$$
\begin{aligned}
M_{f}^{\left(A_{\omega}\right)}(s, \mu, \nu)= & \frac{1}{2 \pi|\nu|}\left|\int_{\omega>0} d \omega \frac{f(\omega)}{\sqrt{\omega}} \exp \left[-i\left(\frac{\mu}{\nu} \omega-\frac{s}{\nu} \log \omega\right)\right]\right|^{2} \\
& +\frac{1}{2 \pi|\nu|}\left|\int_{\omega<0} d \omega \frac{f(\omega)}{\sqrt{|\omega|}} \exp \left[-i\left(\frac{\mu}{\nu} \omega-\frac{s}{\nu} \log |\omega|\right)\right]\right|^{2}
\end{aligned}
$$

$f(\omega)$ being the Fourier transform of the signal $f(t)$.

The tomogram corresponding to the operator

$$
B_{2}^{(A)}=\mu \hat{t}+\nu D
$$

was also computed, namely,

$$
\begin{aligned}
M_{f}^{\left(A_{t}\right)}(s, \mu, \nu)= & \frac{1}{2 \pi|\nu|}\left|\int_{t>0} d t \frac{f(t)}{\sqrt{t}} \exp \left[i\left(\frac{\mu}{\nu} t-\frac{s}{\nu} \log t\right)\right]\right|^{2} \\
& +\frac{1}{2 \pi|\nu|}\left|\int_{t<0} d t \frac{f(t)}{\sqrt{|t|}} \exp \left[i\left(\frac{\mu}{\nu} t-\frac{s}{\nu} \log |t|\right)\right]\right|^{2}
\end{aligned}
$$

The quasidistribution $Q_{f}^{(B)}(\mu, \nu)$ related to the above tomogram is constructed from the affine group. It was discussed in [19]. To compare signal analysis based on the time-scale tomograms and based on wavelets, it is useful to write 
the tomogram $M_{f}^{\left(A_{t}\right)}(s, \mu, \nu)$ in terms of the wavelet transform $W_{f}^{(A)}(s, \tau)$

$$
\begin{aligned}
& M_{f}^{\left(A_{t}\right)}\left(s_{1}, \mu, \nu\right)=\frac{1}{2 \pi\left|\nu N_{h}^{2}\right|} \\
& \times\left\{\left|\int_{t>0, s>0} \frac{d t d \tau d s}{\sqrt{t s} s^{2}} h\left(\frac{t-\tau}{s}\right) W_{f}(s, \tau) \exp \left[i\left(\frac{\mu}{\nu} t-\frac{s_{1}}{\nu} \log t\right)\right]\right|^{2}\right. \\
& \left.+\left|\int_{t<0, s>0} \frac{d t d \tau d s}{\sqrt{|t| s s^{2}}} h\left(\frac{t-\tau}{s}\right) W_{f}(s, \tau) \exp \left[i\left(\frac{\mu}{\nu} t-\frac{s_{1}}{\nu} \log |t|\right)\right]\right|^{2}\right\}
\end{aligned}
$$

The tomographic transform is invertible, that is, the signal may be recovered from the tomogram, namely,

$$
f(t)=\left[\int M_{f}^{\left(A_{t}\right)}(s, \mu, 1) e^{2 i s} d \mu d s\right]^{-1 / 2} \int M_{f}^{\left(A_{t}\right)}(s, \mu, 1) e^{2 i s-i \mu t} d \mu d s
$$

and

$$
\begin{aligned}
f(t) f^{*}\left(t^{\prime}\right)= & \frac{1}{4 \pi^{2}} \int M_{f}^{\left(A_{\omega}\right)}(s, \mu, \nu) \exp \left\{i\left[s-\mu \omega-\nu \omega \frac{t+t^{\prime}}{2}+\omega\left(t-t^{\prime}\right)\right]\right\} \\
& \times|\omega| d \mu d \nu d \omega d s .
\end{aligned}
$$

\section{Meaning of the tomograms}

\subsection{Sampling the phase space}

In the time-frequency space, the tomogram

$$
M_{f}^{(S)}(X, \mu, \nu)=\langle f|\delta(\mu \hat{t}+\nu \hat{\omega}-X)| f\rangle
$$

is the expectation value of an operator delta-function in the state $|f\rangle$. The support of the delta-function in (53) is a line in the time-frequency plane

$$
X=\mu t+\nu \omega
$$

Therefore, $M_{f}^{(S)}(X, \mu, \nu)$ is the marginal distribution of the variable $X$ along this line in the time-frequency space. The line is rotated and rescaled when one changes the parameters $\mu$ and $\nu$. In this way, the whole time-frequency 
space is sampled and the tomographic transform contains all information on the signal.

It is clear that, instead of marginals collected along straight lines on the time-frequency plane, one may use other curves to sample this space. For the tomograms associated to the affine group, one has

$$
M_{f}^{\left(A_{t}\right)}\left(S_{1}, \mu, \nu\right)=\left\langle f\left|\delta\left(\mu \hat{t}+\nu \frac{\hat{t} \hat{\omega}+\hat{\omega} \hat{t}}{2}-S_{1}\right)\right| f\right\rangle
$$

and

$$
M_{f}^{\left(A_{\omega}\right)}\left(S_{2}, \mu, \nu\right)=\left\langle f\left|\delta\left(\mu \hat{\omega}+\nu \frac{\hat{t} \hat{\omega}+\hat{\omega} \hat{t}}{2}-S_{2}\right)\right| f\right\rangle .
$$

The curves in the time-frequency space, defined by

$$
S_{1}=\mu t+\nu t \omega, \quad S_{2}=\mu \omega+\nu t \omega,
$$

are hyperbolas. This becomes clear using the system of coordinates

$$
q=\frac{1}{\sqrt{2}}(t-\omega), \quad p=\frac{1}{\sqrt{2}}(t+\omega) .
$$

In the new coordinates, the curves are

$$
\left(p+\frac{\mu}{\sqrt{2} \nu}\right)^{2}-\left(q-\frac{\mu}{\sqrt{2} \nu}\right)^{2}=\frac{2 S_{1}}{\nu}, \quad\left(p+\frac{\mu}{\sqrt{2} \nu}\right)^{2}-\left(q+\frac{\mu}{\sqrt{2} \nu}\right)^{2}=\frac{2 S_{2}}{\nu},
$$

which are equations for a parametric family of hyperbolas. This means that for the tomograms $M^{\left(A_{t}\right)}\left(S_{1}, \mu, \nu\right)$ and $M^{\left(A_{\omega}\right)}\left(S_{2}, \mu, \nu\right)$ the probability distribution is collected not on straight lines but on hyperbolas. Other generalizations are obvious. One might use marginals on ellipses, parabolas, or on any other algebraic curves.

\subsection{Operator delta-function as a projector density}

While constructing tomograms, the nonnegative operator

$$
\delta(B(\alpha)-X)
$$

plays an essential role, $B(\alpha)$ being an Hermitian operator with nondegenerate continuous spectrum. The random variable $X$ takes values on the spectrum 
of $B(\alpha)$. Considering a set of generalized eigenstates (in $\mathcal{N}^{*}$ ) of $B(\alpha)$, one obtains for the kernel

$$
\left\langle Y|\delta(B(\alpha)-X)| Y^{\prime}\right\rangle=\delta\left(Y^{\prime}-X\right) \delta\left(Y-Y^{\prime}\right)=\langle Y \mid X\rangle\left\langle X \mid Y^{\prime}\right\rangle .
$$

Therefore, we may identify $\delta(B(\alpha)-X)$ with the projector $|X\rangle\langle X|$

$$
\delta(B(\alpha)-X)=|X\rangle\langle X|=P_{X} .
$$

From this, it follows

$$
M_{f}^{(B)}=\langle f|\delta(B(\alpha)-X)| f\rangle=\langle f \mid X\rangle\langle X \mid f\rangle=|\langle X \mid f\rangle|^{2}
$$

displaying the positivity of the tomogram. This means that there is always a basis in $\mathcal{N}^{*}$ such that, by projecting on this basis, the tomogram is the product of two complex conjugate functions. By a unitary transformation $S$, $B(\alpha)$ may be transformed to

$$
S B(\alpha) S^{\dagger}=B^{\prime}(\alpha)
$$

Then if $\{|Z\rangle\}$ is the set of (generalized) eigenvectors of $B^{\prime}(\alpha),\left\{S^{\dagger}|Z\rangle\right\}$ is a set of eigenvectors for $B$. Therefore,

$$
M_{f}^{(B)}(Z)=\langle f|\delta(B(\alpha)-Z)| f\rangle=|\langle Z|S| f\rangle|^{2}=\left\langle f\left|S^{\dagger}\right| Z\right\rangle\langle Z|S| f\rangle .
$$

In this case, the operator $U(\alpha)$ in the general scheme described in Sect. 2 would be

$$
U(\alpha)=S^{\dagger}|Z\rangle\langle Z| S,
$$

which, in this case, is not represented as an exponent of an operator. The form (66), with the presence of a projector operator $P_{Z}=|Z\rangle\langle Z|$, also shows the relation of the tomograms to transforms of the Husimi-Kano type. Notice, however, that, for example, the time-frequency Husimi-Kano transform

$$
Q_{f}(t, \omega)=|\langle\beta \mid f\rangle|^{2}, \quad \beta=\frac{t+i \omega}{\sqrt{2}},
$$

where $|\beta\rangle$ is a coherent state, does not describe a joint probability distribution in the time-frequency plane, because time and frequency do not commute and a joint probability distribution of two noncommuting observables cannot exist due to the uncertainty relation. Therefore, the correct way to interpret the Husimi-Kano quasidistribution is not as a joint time-frequency probability but as a unitarily transformed tomogram. 


\section{Discrete Spectrum}

In the case where the operator $B(\alpha)$ has a discrete spectrum, one uses a Kronecker delta-function $\delta_{K}(B(\alpha)-n)$ and associates to the operator the Fourier integral on the circle. For example, for the number operator

$$
\delta_{K}\left(a^{\dagger} a-n\right)=\frac{1}{2 \pi} \int_{0}^{2 \pi} e^{i \varphi\left(a^{\dagger} a-n\right)} d \varphi ; \quad n=0,1,2,3 \ldots,
$$

and the matrix elements of this operator in the Fock basis $|m\rangle$ are

$$
\left\langle m^{\prime}\left|\delta_{K}\left(a^{\dagger} a-n\right)\right| m\right\rangle=\delta_{m^{\prime} m} \delta_{m n} ; \quad m, m^{\prime}=0,1,2,3 \ldots,
$$

which equal the matrix elements of the projector

$$
P_{n}=|n\rangle\langle n| \text {. }
$$

This means that, also in the discrete-spectrum case, the Kronecker deltafunction of the operator $B(\alpha)$ is reduced to a projector. The tomogram associated to the Wigner-Ville function by this method is given by the socalled photon-number tomography [25] [26] [27], i.e.,

$$
\begin{aligned}
& w(n, \beta)=\left\langle f\left|D^{\dagger}(\beta)\right| n\right\rangle\langle n|D(\beta)| f\rangle \\
= & \frac{1}{\sqrt{\pi} 2^{n} n !}\left|\int d t e^{-\left(t^{2} / 2\right)+i \sqrt{2} t \operatorname{Im} \beta} H_{n}(t) f(t+\sqrt{2} \operatorname{Re} \beta)\right|^{2},
\end{aligned}
$$

where the complex number $\beta$ is a linear combination of the parameters $\mu$ and $\nu$.

One may also construct a tomogram using a Dirac delta-function of the same operator $B(\alpha)$

$$
\begin{aligned}
& M(X, \Omega, \tau)=\int f\left(t^{\prime}+k \tau\right) f^{*}\left(t^{\prime \prime}-k \tau\right) e^{-i k(X+\pi / 2)} \frac{i}{2 \pi \sin (k \pi / 2)} \\
& \times e^{-i k \Omega\left(t^{\prime}+t^{\prime \prime}\right)} \exp \left[-\frac{i}{2} \cot \frac{k \pi}{2}\left(2 t^{2}+t^{\prime 2}+t^{\prime \prime 2}\right)+\frac{i t}{\sin (k \pi / 2)}\left(t^{\prime}+t^{\prime \prime}\right)\right] d k d t^{\prime} d t^{\prime \prime} d t .
\end{aligned}
$$

The inverse of the transform reads

$f(t) f^{*}\left(t^{\prime}\right)=\frac{1}{\pi} \int M\left(X-\frac{\pi}{2}, \Omega, \frac{t^{\prime}-t}{2}\right) \exp \left[-i \Omega\left(t+t^{\prime}\right)+i\left(X+\frac{\pi}{2}\right)\right] d \Omega d X$. 
In this case the Dirac delta-function of $B(\alpha)-X$ is not reduced to a projector density. The tomogram (72) corresponds to marginals collected from shifted circles in the classical phase space.

\section{Remarks and conclusions}

1 - The main result in this work is the formulation of an unified view for some linear and nonlinear transforms through the operator formulation developed in Sect. 2. The formulation emphasizes the basic unity of these transforms, which are related by explicit formulas. Nevertheless, for each particular application, one type of transform may be more convenient than the others. In particular, when non-ambiguous joint information on noncommutative observable planes is desired, tomograms seem to be the most competitive type of transforms.

The formulation applies both to unitary operators or nonunitary ones of the form (13). It is also an appropriate framework to construct new transforms once a particular aspect of the signal is defined and this is expressed through the corresponding operator set.

2 - The operators $U(\vec{\alpha})$ may belong to group representations or be operators of a deformed group. In the cases we have considered in detail, the operators belong to the Lie algebra of the group $\operatorname{ISp}(2, R)$, which has the following six generators:

$$
L_{1}=\hat{t}, \quad L_{2}=\hat{\omega}, \quad L_{3}=1, \quad L_{4}=\hat{t}^{2}, \quad L_{5}=\hat{\omega}^{2}, \quad L_{6}=\frac{1}{2}(\hat{t} \hat{\omega}+\hat{\omega} \hat{t}) .
$$

When it is unitary, the operator $U(\vec{\mu})$ may be considered to be an evolution operator associated to an Hamiltonian operator formed from the generators of the group. Quasidistributions evolve the state $\mid f>$ and project the evolved state on the initial condition. On the other hand, the tomograms collect the probability density on a family of lines in phase space. In the cases that were studied, hyperbolas, straight lines and circles were considered. Other types of tomograms might be considered, for example, those corresponding to parabolas, that is, $X=\mu t+\nu \omega^{2} \mathrm{~A}$ similar construction might be done for other groups and other algebraic structures like quantum groups. 


\section{References}

[1] A. D. Poularikas (ed.); The Transforms and Applications Handbook, CRC Press \& IEEE Press, Boca Raton, Florida (1996).

[2] K.-B. Wolf; Integral Transforms in Science and Engineering, Plenum Press, New York (1979).

[3] J. B. J. Fourier; Théorie Analytique de la Chaleur, in: G. Darbous (ed.), Oeuvres de Fourier, Gauthiers-Villars, Paris (1888), Tome premier.

[4] J. M. Combes, A. Grossmann, and Ph. Tchamitchian (eds.); Wavelets, Springer, Berlin (1990), 2nd edition.

[5] I. Daubechies; "The wavelet transform: time-frequency localization and signal analysis," IEEE Trans. Inform. Theory, 36, No. 5 (1990) 9611005 .

[6] C. K. Chui (ed.); Wavelets: A Tutorial. Theory and Applications, Academic, Boston (1992), Vol. 2.

[7] E. Wigner; "On the quantum correction for thermodynamic equilibrium," Phys. Rev., 40 (1932) 749-759.

[8] J. Ville; "Théorie et applications de la notion de signal analytique," Cables et Transmission, 2 A (1948) 61-74.

[9] L. Cohen; "Generalized phase-space distribution functions," J. Math. Phys. 7 (1966) 781-806.

[10] S. Qian and D. Chen; Joint time-frequency analysis, Prentice-Hall, Englewood Cliffs, N. J. (1995).

[11] J. C. Woods and D. T. Barry; "Linear signal synthesis using the RadonWigner transform," IEEE Trans. Signal Process. 42 (1994) 2105-2111.

[12] S. Granieri, W. D. Furlan, G. Saavedra, and P. Andrés; "Radon-Wigner display: a compact optical implementation with a single varifocal lens," Appl. Opt. 36 (1997) 8363-8369. 
[13] V. I. Man'ko and R. Vilela Mendes; "Noncommutative time-frequency tomography," Phys. Lett. A, 263 (1999) 53-59.

[14] R. J. Glauber; "Coherent and incoherent states of the radiation fields," Phys. Rev. 131 (1963) 2766-2788; "Photon correlations," Phys. Rev. Lett. 10 (1963) 84-86.

[15] E. C. G. Sudarshan, "Equivalence of semiclassical and quantummechanical descriptions of statistical light beams," Phys. Rev. Lett. 10 (1963) 277-279.

[16] K. Husimi; "Some formal properties of the density matrix," Proc. Phys. Mat. Soc. Jpn, 22 (1940) 264-314.

[17] Y. Kano; "A new phase-space distribution function in the statistical theory of the electromagnetic field," J. Math. Phys. 6 (1965) 1913-1915.

[18] K. E. Cahill and R. J. Glauber; "Density operators and quasiprobability distributions," Phys. Rev. 177 (1969) 1882-1902.

[19] J. Bertrand and P. Bertrand; "A class of affine Wigner functions with extended covariance properties," J. Math. Phys., 33 (1992) 2515-2527.

[20] P. Goncalvés and R. G. Baraniuk; "A pseudo-Bertrand distribution for time-scale analysis," IEEE Signal Process. Lett. 3 (1996) 82-84.

[21] P. Flandrin and P. Goncalvés; "From wavelets to time-scale energy distributions," in: L. L. Schumaker and G. Webb (eds.), Recent Advances in Wavelet Analysis, Academic Press (1994), pp. 309-334.

[22] L. Cohen; "The scale representation," IEEE Trans. Signal Process. 41 (1993) 3275-3292.

[23] L. Cohen; "Time-frequency distributions. — A review," Proc. IEEE 77 (1989) 941-981.

[24] V. V. Dodonov and V. I. Man'ko; in Invariants and Evolution of Nonstationary Quantum Systems, Proceedings of the Lebedev Physics Institute v. 183, Nova Science, Commack, NY 1989. 
[25] S. Mancini, P. Tombesi and V. I. Man'ko; "Density matrix from photon number tomography," Europhys. Lett. 37 (1997) 79-83.

[26] S. Wallentowitz and W. Vogel; "Unbalanced homodyning for quantum state measurements," Phys. Rev. A 53 (1996) 4528-4533.

[27] K. Banaszek and K. Wodkiewicz; "Direct probing of quantum phase space by photon counting", Phys. Rev. Lett. 76 (1996) 4344-4347. 